\title{
Inhibition of Fusarium oxysporum f. sp. nicotianae Growth by Phenylpropanoid Pathway Intermediates
}

\author{
Timothy E. Shull ${ }^{1}$, Jasmina Kurepa ${ }^{1}$, Robert D. Miller ${ }^{1}$, Natalia Martinez-Ochoa ${ }^{10}{ }^{1,2 *}$, and Jan A. Smalle (D) ${ }^{1,2 *}$ \\ ${ }^{1}$ Department of Plant and Soil Sciences, University of Kentucky, Lexington, KY 40546-0236, USA \\ ${ }^{2}$ KTRDC, University of Kentucky, Lexington, KY 40546-0236, USA
}

(Received on August 24, 2020; Revised on September 14, 2020; Accepted on September 14, 2020)

Fusarium wilt in tobacco caused by the fungus Fusarium oxysporum f. sp. nicotianae is a disease-management challenge worldwide, as there are few effective and environmentally benign chemical agents for its control. This challenge results in substantial losses in both the quality and yield of tobacco products. Based on an in vitro analysis of the effects of different phenylpropanoid intermediates, we found that the early intermediates trans-cinnamic acid and para-coumaric acid effectively inhibit the mycelial growth of $F$. oxysporum f. sp. nicotianae strain FW316F, whereas the downstream intermediates quercetin and caffeic acid exhibit no fungicidal properties. Therefore, our in vitro screen suggests that trans-cinnamic acid and para-coumaric acid are promising chemical agents and natural lead compounds for the suppression of $F$. oxysporum $f$. sp. nicotianae growth.

Keywords: fungicidal, Fusarium oxysporum, para-coumaric acid, tobacco, trans-cinnamic acid

\footnotetext{
*Co-corresponding authors.

Jan A. Smalle

Phone) +1-859-257-3677, Fax) +1-859-323-1077

E-mail)jsmalle@uky.edu

Natalia Martinez-Ochoa

Phone) +1-859-257-3633, Fax) +1-859-323-1077

E-mail) Natalia.Martinez@uky.edu

ORCID

Jan A. Smalle

https://orcid.org/0000-0002-6067-4619

Natalia Martinez-Ochoa

https://orcid.org/0000-0002-2280-8997

(c) This is an Open Access article distributed under the terms of the Creative Commons Attribution Non-Commercial License (http:// creativecommons.org/licenses/by-nc/4.0) which permits unrestricted noncommercial use, distribution, and reproduction in any medium, provided the original work is properly cited.
}

Articles can be freely viewed online at www.ppjonline.org.
Handling Editor : Junhyun Jeon

Nonpathogenic and pathogenic strains of the soil-borne fungus Fusarium oxysporum are ubiquitous in agricultural soils worldwide, and pathogenic sub-specific strains (i.e., formae speciales; f. sp.) impact a number of economically important crops (Gordon, 2017). F. oxysporum $\mathrm{f}$. sp. nicotianae (FON) is the causal agent of Fusarium wilt disease (FW) in tobacco, which causes chlorosis, wilting and necrosis of tobacco leaves that leads to stunted growth and death. FON affects the global tobacco crop due to high seed transmissibility and its ability to survive in soil without a host plant for years (LaMondia, 2015).

To date, the use of FW-resistant cultivars is the most effective FW management strategy. However, breeding programs are time-consuming and costly, and resistant cultivars are not entirely immune to FW infection under high disease pressure (LaMondia, 1995). Therefore, FW chemical management, through pre-planting soil fumigation with methyl bromide or chloropicrin, is commonplace in many plant production systems (Duniway, 2002). However, methyl bromide is now recognized as a Class I ozone-depleting substance and its use has been phased out (UNEP Ozone Secretariat, 2020) and chloropicrin is a strong irritant that decreases tobacco quality and often does not appreciably decrease disease incidence (Fisher et al., 2018; LaMondia, 2015; Schneider et al., 2003; Xie et al., 2015). Other classes of fungicides such as succinate dehydrogenase inhibitors and demethylation inhibitors have shown promise for the control of soil-borne fungal pathogens (Masiello et al., 2019; Miller et al., 2020) but these compounds have not been systematically tested on Fusarium species. Moreover, the wide-spread use of fungicides with single-site mechanisms of action necessitates 
a diverse set of chemical management tools to slow down the evolution of fungicide-resistant pathogens (Avenot and Michailides, 2010; Lucas et al., 2015). Therefore, there is a continuing demand for cost-effective and environmentally benign chemicals for FW disease management in tobacco and other cropping systems.

The demand for novel fungicides and ecologically non-disruptive FW treatments led to the expansion of research focused on the identification of natural products which can control FW in various plant systems (Bubici et al., 2019; Hazir et al., 2017; Steinkellner and Mammerler, 2007; Wu et al., 2008). This research showed that plant secondary metabolites synthesized by the phenylpropanoid pathway (PPP) can inhibit the growth of some $F$. oxysporum strains (Steinkellner and Mammerler, 2007). The PPP begins with the conversion of phenylalanine to trans-cinnamic acid ( $t$-CA) by phenylalanine ammonia-lyase (Vogt, 2010). $t$-CA is processed by cinnamic acid 4-hydrolase into para-coumaric acid ( $p$-CA). $p$-CA is converted into para-coumaroyl-CoA, a major branch-point metabolite of the PPP, which is a precursor of various compounds such as caffeic acid and quercetin (Fig. 1) (Vogt, 2010).

Phenylpropanoids (PPs) have many beneficial properties which have attracted intense research interest (Neelan et al., 2019). Analyses of the fungicidal properties of PPs emphasized the research centered on $t$-CA (Guzman, 2014). Currently, there is no consensus on $t$-CA fungicidal activity even in a single species. For example, studies conducted on $F$. oxysporum indicate that $t$-CA can both promote and inhibit mycelial growth (Wu et al., 2008; Ye et al., 2004; Zhao et al., 2018), and the current hypothesis is that the growth inhibitory activity of $t$-CA on FW-causing pathogens varies due to the high physiological variability of F. oxysporum subspecies (Bock et al., 2014; Hao et al., 2010; Hazir et al., 2017; Morales et al., 2017; Nirmaladevi et al., 2016; Wu et al., 2008). The impact of other PPs on FW-causing pathogens has also been analyzed, although not in great detail. For example, $p$-CA and caffeic acid have both been shown to decrease the sporulation and germination of Fusarium oxysporum f. sp. niveum (Hao et al., 2010).

Because the wide-spread interest in the identification of environmentally benign FON control is in stark contrast with the lack of information on the anti-FON activity of natural products, here we lay the groundwork for research into the efficacy of PPP intermediates in controlling FON growth, and demonstrate that $t$-CA and its direct downstream derivative, $p$-CA, effectively mitigate the growth of the FON strain FW316F.

FON strain FW316F was single-spore purified from the stem of a TN 97 tobacco plant growing at the University of Kentucky-Spindletop Farm in Lexington, and identified as FON based on conidia morphology (Leslie et al., 2006). For the preparation of the FON inoculum used in all experiments described in this study, FON was grown for two weeks in the dark at $25^{\circ} \mathrm{C}$ on potato dextrose agar (PDA; Difco, Detroit, MI, USA), scrapped into $15 \mathrm{ml}$ of distilled water, vortexed and briefly centrifuged to pellet mycelial debris. To make the final inoculum, the supernatant was diluted until the solution absorbance at $600 \mathrm{~nm}$ was $0.1 \pm 0.02$. An aliquot of each inoculum was transferred onto a hemocytometer (C-chip DHC-N01, SKC Inc., Cheonan, Korea), and micrographs were captured using an Olympus SZX12 microscope. The microconidia/ml was determined to be $257,857 \pm 65,183$ (mean \pm standard deviation, $n=6$ ) using the open-source program ImageJ (http://www.imagej.net).

Considering that previous studies demonstrated the antifungal activity of $t$-CA (Guzman, 2014), we initiated our analyses with this compound. To that end, we prepared a control PDA plate (no additives), test plates (with $0.1 \mathrm{mM}$ and $1 \mathrm{mM} t$-CA; $t$-CA obtained from Sigma-Aldrich, St. Louis, MO, USA), a solvent control plate (with $0.55 \%$ dimethyl sulfoxide [DMSO]) and a positive control plate (with $0.2 \%$ of the commercial fungicide Cleary 3336F). FON inoculum $(100 \mu \mathrm{l})$ was spread onto the prepared plates, which were then incubated in the dark for four days

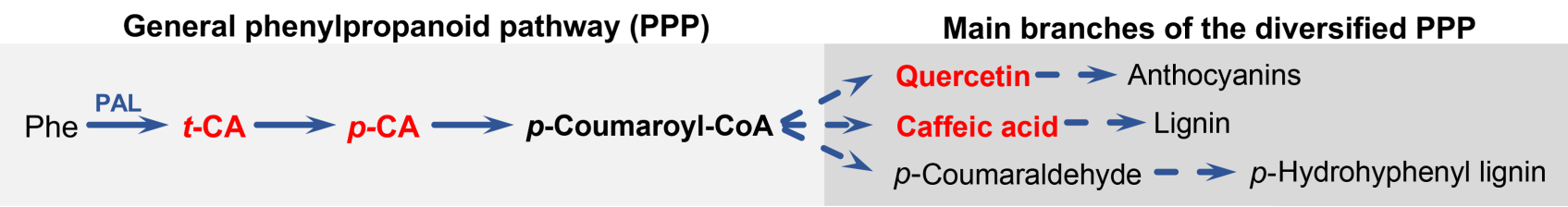

Fig. 1. Simplified scheme of the phenylpropanoid biosynthetic pathway. The phenylpropanoid biosynthetic pathway (PPP) channels carbon from L-phenylalanine (Phe) to a large number of polyphenols. The general PPP beings with Phe which is converted to trans-cinnamic acid ( $t$-CA) by the enzyme phenylalanine ammonia lyase. $t$-CA is then reduced to para-coumaric acid ( $p$-CA) which is activated to yield $p$-coumaroyl-CoA. $p$ (ara)-Coumaroyl-CoA is the branch-point metabolite of all flavonoid and non-flavonoid polyphenols. The compounds tested in this study are highlighted in red. 


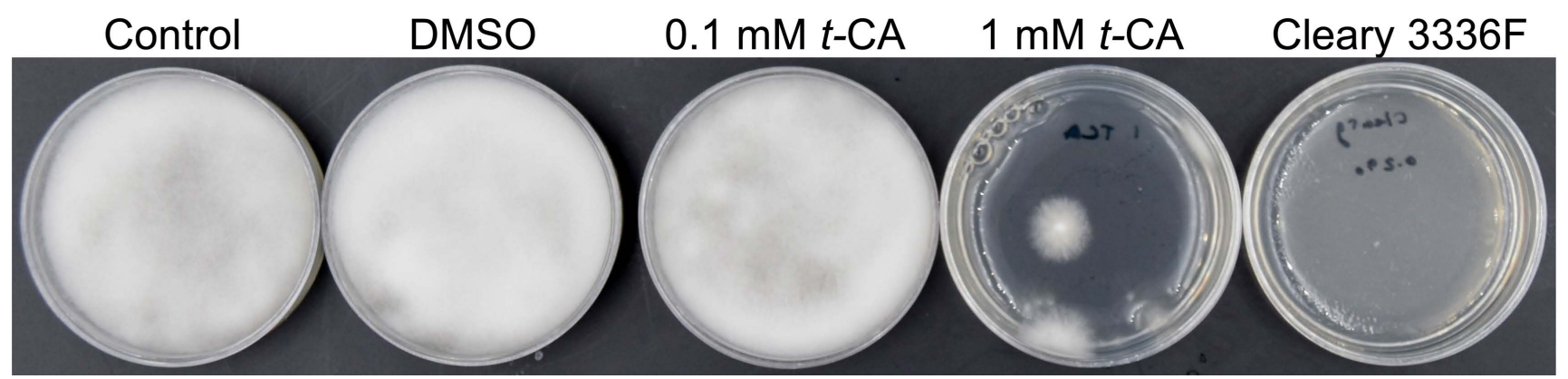

Fig. 2. Trans-cinnamic acid ( $t$-CA) inhibits the growth of Fusarium oxysporum f. sp. nicotianae. Fusarium oxysporum f. sp. nicotianae inoculum was spread on potato dextrose agar plates without additives (control), with $0.5 \%$ dimethyl sulfoxide (DMSO; as a solvent control for the $1 \mathrm{mM} t$-CA treatment), with $0.1 \mathrm{mM}$ or $1 \mathrm{mM} t$-CA, and with $0.2 \%$ of the commercial fungicide Cleary $3336 \mathrm{~F}$. Plates were incubated in the dark at room temperature for 4 days and photographed.

at room temperature and evaluated (Fig. 2). As expected, the $0.2 \%$ Cleary $3336 \mathrm{~F}$ treatment completely inhibited FON growth. Mycelia grew equally on plates without additives and plates with the solvent control, suggesting that the $t$-CA solvent has no effect on fungal growth at the tested doses. The $t$-CA dose-response plates revealed that whereas $0.1 \mathrm{mM} t$-CA had no antifungal properties, $1 \mathrm{mM}$ $t$-CA led to nearly complete inhibition of mycelial growth, proving to be almost as effective as the $0.2 \%$ Cleary $3336 \mathrm{~F}$ treatment (Fig. 2). Therefore, we concluded that $t$-CA has dose-dependent antifungal activity against the FON strain FW316F.

Because $t$-CA is the first compound synthesized in the PPP, we next asked if $t$-CA itself or if a downstream metabolite is responsible for the observed fungicidal activity. To that end, we tested three compounds: (1) $p$-CA, a direct downstream derivative of $t$-CA, (2) the late downstream intermediate caffeic acid, which is a lignin precursor, and (3) quercetin, a late intermediate that belongs to the flavanol class of the PP compounds named flavonoids. To compare
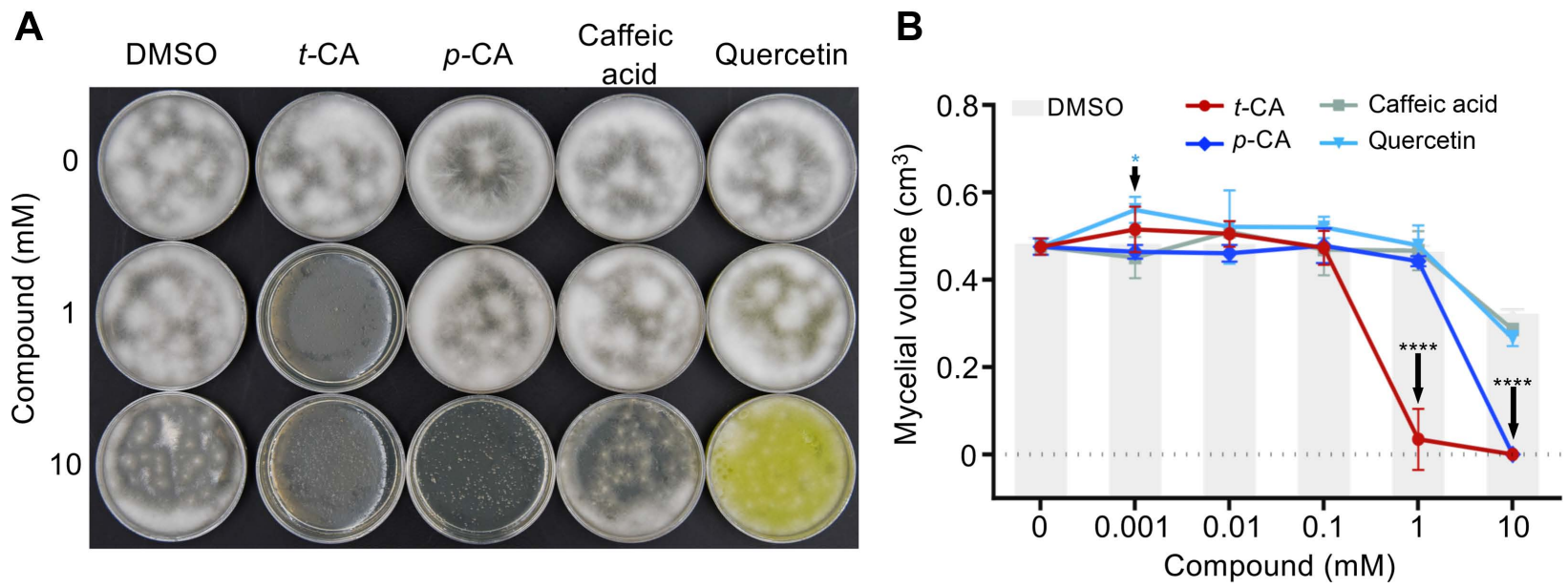

Fig. 3. Effects of phenylpropanoids on Fusarium oxysporum f. sp. nicotianae (FON) growth. (A) Trans-cinnamic acid ( $t$-CA) and para-coumaric acid ( $p$-CA) inhibit the growth of FON. One hundred microliters of FON inoculum was spread on potato dextrose agar (PDA) media containing the denoted concentrations of compounds and incubated in the dark for 4 days. Quercetin is a yellow-colored plant pigment and at higher concentrations, it stains the media. (B) Quantification of the growth-inhibitory effects of phenylpropanoids. Solvent controls (dimethyl sulfoxide [DMSO]; gray bars) contained the concentration of DMSO used to prepare the compound solution at the labeled tick-mark (e.g., for $0.001 \mathrm{mM}, 0.0005 \%$ DMSO was used as a control; for $0.01 \mathrm{mM}, 0.005 \% \mathrm{DMSO}$ ). The mycelium from each plate was scrapped into tubes containing $1 \mathrm{ml}$ of water after four days of growth on the PDA media. The mycelial suspension was centrifuged at $21,330 \times g$ for $5 \mathrm{~min}$.. The mycelial pellet was then leveled, photographed and the mycelial volume was measured. Data are presented as mean $\pm \mathrm{SD}(n=3) . * P<0.05, * * * * P<0.0001$. $p$-values on the graph are post-hoc comparisons of the DMSO solvent controls to the point(s) indicated by the black arrows. The light blue asterisk indicates the comparison between the $0.001 \mathrm{mM}$ quercetin treatment and the DMSO control. 
the FON-inhibitory capacity of these three downstream compounds to $t$-CA, we prepared PDA media supplemented with $t$-CA, $p$-CA (Sigma-Aldrich), caffeic acid (3,4-dihydroxy-cinnamic acid, Sigma-Aldrich), and quercetin $\left(5,7,3^{\prime}, 4^{\prime}\right.$-flavon-3-ol, Sigma-Aldrich) at concentrations of $0.001 \mathrm{mM}, 0.01 \mathrm{mM}, 0.1 \mathrm{mM}, 1 \mathrm{mM}$ and $10 \mathrm{mM}$. The FON inoculum $(100 \mu \mathrm{l})$ was spread on the plates, and plates were incubated in the darkness at room temperature. After four days of incubation, mycelial growth was inhibited on media containing $1 \mathrm{mM}$ and $10 \mathrm{mM} t$-CA as well as on plates containing $10 \mathrm{mM} p$-CA (Fig. $3 \mathrm{~A}$ ).

To quantify the inhibitory effect of the tested PPs, mycelia were scrapped into $1 \mathrm{ml}$ of water, pelleted by centrifugation, leveled with a spatula, photographed and the mycelial volume was measured using ImageJ software. After three independent replicates, the collected data $(n=3)$ were analyzed using GraphPad Prism 6.0 (two-way ANOVA followed by Tukey's multiple comparisons post-hoc test, GraphPad Software Inc., San Diego, CA, USA). $P \leq 0.05$ from the post-hoc tests were considered significant and are marked on the graphs. As previously observed (Fig. 2 ), there was no significant difference in growth between the control plates and most of the solvent (DMSO) control plates (Fig. 3B). The exception was the DMSO control for the $10 \mathrm{mM}$ treatments, which led to an $\sim 34 \%$ reduction in mycelial growth. However, $10 \mathrm{mM} t$-CA and $10 \mathrm{mM} p$-CA completely inhibited mycelial growth, indicating that at this dose, the effect of the test compound was stronger than the effect of the solvent. Quantitative analyses showed that the inhibition of mycelial growth was statistically significant when compared with the solvent control in the $1 \mathrm{mM} t$-CA, $10 \mathrm{mM} t$-CA, and $10 \mathrm{mM} p$-CA treatments (Fig. 3B). Conversely, caffeic acid treatments were not significantly different from the control at any tested doses. Quercetin also did not show any anti-FON activity. In fact, the lowest tested dose of quercetin $(0.001 \mathrm{mM})$ led to a slight increase in mycelial volume (Fig. 3B). Although the exact mechanism of this growth promotion was not further analyzed, quercetin is known to be a strong antioxidant (Xu et al., 2019), and as such, it may have improved mycelial growth by
A

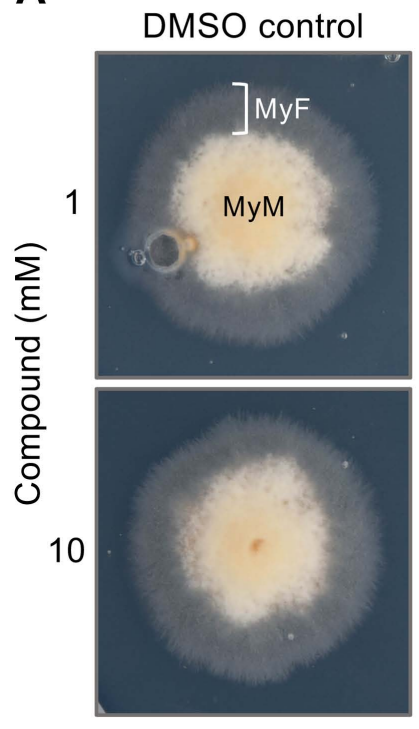

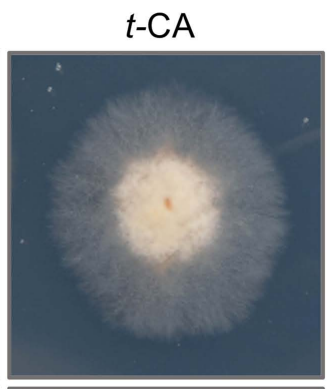

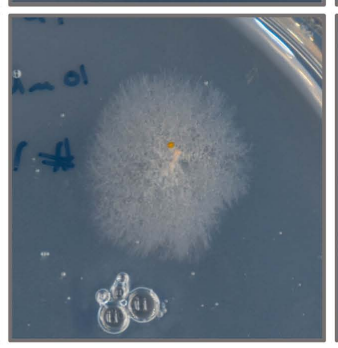

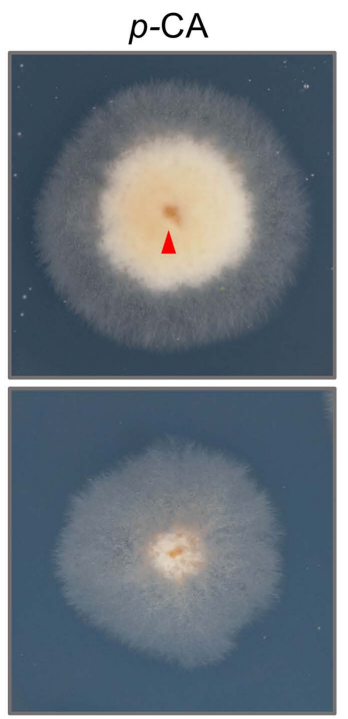

B

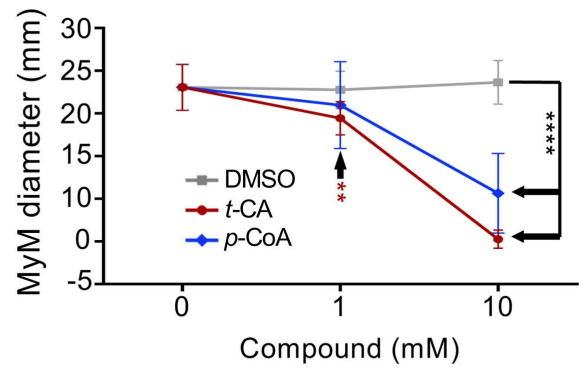

C

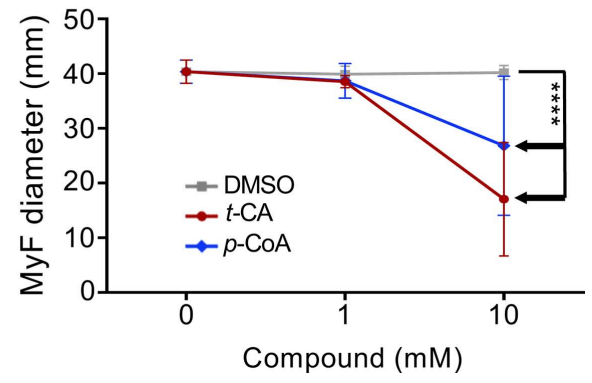

Fig. 4. Trans-cinnamic acid ( $t$-CA) and para-coumaric acid ( $p$-CA) inhibit Fusarium oxysporum f. sp. nicotianae (FON) mycelial growth originating from tobacco seed surface transmission. (A) $t$-CA and $p$-CA decrease mycelial front diameter originating from FON-contaminated KT97 seeds. Tobacco seeds of the FON-susceptible variety KT97 were sterilized and suspended in $1 \mathrm{ml}$ of sterile distilled water and $100 \mu \mathrm{l}$ of FON inoculum was added to the solution. $t$-CA, $p$-CA or an equivalent amount of dimethyl sulfoxide (DMSO) were then added to the seed/FON mix at the denoted concentrations. After briefly vortexing the mix, the seeds were sown on tobacco growth media. Plates were then incubated for 4 days in a growth chamber under continuous light after which they were photographed. Photographs are $40 \times 40 \mathrm{~mm}$. Red arrowhead points to a tobacco seed. MyM, mycelial mat; MyF, mycelial front. (B, C) $t$-CA and $p$-CA decrease mycelial mat diameter originating from FON contaminated KT97 seeds. Experiment was performed as in A and data were gathered from photographs using ImageJ. Data presented are mean $\pm \mathrm{SD}(n=15)$. $* * * * P 0.0001 . P$-values on the graph are the result of post-hoc comparisons of the DMSO solvent controls to the point(s) indicated by the black arrows. The red asterisks in B indicate the comparison between the $1 \mathrm{mM} t$-CA treatment and the DMSO control. 
reducing oxidative stress. Taken together, our data suggest that the simple, early PPP intermediates $t$-CA and $p$-CA effectivity inhibit the growth of FON strain FW316F, and of the tested intermediates, $t$-CA has the highest potency.

Due to the high transmissibility of FON in seeds (LaMondia, 2015), we opted to test if $t$-CA and $p$-CA can be used to inhibit FON spread stemming from surface-contaminated tobacco seeds. To that end, we aliquoted $10 \pm 2$ $\mathrm{mg}$ of surface-sterilized seeds of the FON-susceptible tobacco variety TN 97 (FW. Rickard Seeds, Inc., Winchester, $\mathrm{KY}$, USA) into tubes, added $100 \mu \mathrm{l}$ of FON inoculum to each tube and then briefly vortexed the mix. A volume of $t$-CA or $p$-CA DMSO stock solution was added to the tubes to attain concentrations of $1 \mathrm{mM}$ or $10 \mathrm{mM}$. For solvent controls, an equivalent volume of DMSO was added to the seed/FON inoculum mix. The treated FON-contaminated seeds were sown on full-strength Murashige and Skoog media containing 3\% sucrose and $0.7 \%$ Phytoagar (PhytoTech, Shawnee Mission, KS, USA) and incubated for 4 days in a growth chamber under continuous light (17-Watt fluorescent lights, color temperature 4100) at a constant temperature $\left(28^{\circ} \mathrm{C}\right)$. The control seeds developed thick, opaque mycelial mats (MyM) and thinner, translucent mycelial fronts $(\mathrm{MyF})$ around the periphery of the MyM, whereas the MyM around the seeds treated with $1 \mathrm{mM}$ and $10 \mathrm{mM} t$-CA and $10 \mathrm{mM} p$-CA was reduced in size (Fig. $4 \mathrm{~A})$.

To quantify the impact of the $t$-CA and $p$-CA seed treatments on FON seed-surface transmission, we measured the $\mathrm{MyF}$ and MyM diameters using ImageJ software (Fig. 4B and $\mathrm{C}$ ). After three independent replicates, the collected data $(n=15)$ were analyzed using GraphPad Prism 6.0. Two-way ANOVA was used to compare means between groups, and Tukey's multiple comparisons was used as a post-hoc test. $P \geq 0.05$ from the post-hoc tests were considered significant. Compared to the DMSO treatments, 1 $\mathrm{mM}$ and $10 \mathrm{mM} t$-CA led to significant, dose-dependent reductions in MyM and MyF diameters. Similar to the in vitro growth experiments (Fig. 3), $p$-CA was less effective than $t$-CA in decreasing the spread of FON from infected seeds, as indicated by the decrease in MyM and MyF diameter only at the highest tested dose (Fig. 4B and C). We observed complete inhibition of mycelial growth around infected seeds in $40 \% \pm 2 \%$ of cases when treated with 10 $\mathrm{mM} t$-CA and in $20 \% \pm 2 \%$ of $10 \mathrm{mM} p$-CA treatments. These results suggest that compared to $t$-CA, $p$-CA is approximately half as effective at inhibiting FON mycelial growth. Collectively, our results indicate that the PP intermediates $t$-CA and $p$-CA are both promising natural products for the control of FON spread in tobacco produc- tion systems, and that $t$-CA is the most effective PP tested for the inhibition of the mycelial growth of FON strain FW316F.

Natural products have been a strong lead in the discovery and development of novel fungicides (Daoubi et al., 2005; Li et al., 2018; Musso et al., 2012). In the present study, we demonstrate that both $t$-CA and $p$-CA are potentially useful anti-FON agents and possible lead compounds for the development of new synthetic fungicides. More research is required to determine if the antifungal capacity of $t$-CA and $p$-CA generalizes to all FON strains. Of equal importance is the testing of the FON-inhibitory capacity of PPP intermediates on susceptible tobacco plants exposed to FON. The use of PPP derivatives to mitigate FON infection has potential limitations. For example, $t$-CA and $p$-CA are poorly soluble in aqueous solutions (Mota et al., 2008), which complicates the creation of formulations for field applications. Furthermore, using plant metabolites to control FON may cause off-target effects on plant growth and development. However, the strong fungicidal activity of $t$-CA and $p$-CA, their availability, and low environmental risk give a strong impetus for a detailed understanding of the mechanism of their FON growth-inhibitory activity, which in turn may aid in the development of novel environmentally friendly fungicides.

\section{Conflicts of Interest}

No potential conflict of interest relevant to this article was reported.

\section{Acknowledgments}

This work was supported by the Kentucky Tobacco Research and Development Center and a Phillip Morris International grant in 2017.

\section{References}

Avenot, H. F. and Michailides, T. J. 2010. Progress in understanding molecular mechanisms and evolution of resistance to succinate dehydrogenase inhibiting (SDHI) fungicides in phytopathogenic fungi. Crop Prot. 29:643-651.

Bock, C. H., Shapiro-Ilan, D. I., Wedge, D. E. and Cantrell, C. L. 2014. Identification of the antifungal compound, transcinnamic acid, produced by Photorhabdus luminescens, a potential biopesticide against pecan scab. J. Pest Sci. 87:155162.

Bubici, G., Kaushal, M., Prigigallo, M. I., Gómez-Lama Cabanás, C. and Mercado-Blanco, J. 2019. Biological control agents against Fusarium wilt of banana. Front. Microbiol. 10:616. 
Daoubi, M., Hernández-Galán, R., Benharref, A. and Collado, I. G. 2005. Screening study of lead compounds for natural product-based fungicides: antifungal activity and biotransformation of $6 \alpha, 7 \alpha$-dihydroxy- $\beta$-himachalene by Botrytis cinerea. J. Agric. Food Chem. 53:6673-6677.

Duniway, J. M. 2002. Status of chemical alternatives to methyl bromide for pre-plant fumigation of soil. Phytopathology 92:1337-1343.

Fisher, C., Pearce, B. and Kinney, J. 2018. Chloropicrin fumigation to control fusarium wilt of burley tobacco in Kentucky. URL https://www.coresta.org/sites/default/files/abstracts/2018_TWC48_Fisher.pdf. [14 October 2020].

Gordon, T. R. 2017. Fusarium oxysporum and the fusarium wilt syndrome. Annu. Rev. Phytopathol. 55:23-39.

Guzman, J. D. 2014. Natural cinnamic acids, synthetic derivatives and hybrids with antimicrobial activity. Molecules 19:1929219349.

Hao, W.-Y., Ren, L.-X., Ran, W. and Shen, Q.-R. 2010. Allelopathic effects of root exudates from watermelon and rice plants on Fusarium oxysporum f.sp. niveum. Plant Soil 336:485-497.

Hazir, S., Shapiro-Ilan, D. I., Bock, C. H. and Leite, L. G. 2017. Trans-cinnamic acid and Xenorhabdus szentirmaii metabolites synergize the potency of some commercial fungicides. $J$. Invertebr. Pathol. 145:1-8.

LaMondia, J. A. 1995. Influence of resistant tobacco and tobacco cyst nematodes on root infection and secondary inoculum of Fusarium oxysporum f. sp. nicotiana. Plant Dis. 79:337-340.

LaMondia, J. A. 2015. Fusarium wilt of tobacco. Crop Prot. 73:73-77.

Leslie, J. F., Summerell, B. A. and Bullck, S. 2006. The Fusarium laboratory manual. Blackwell Publishing, Ames, IA, USA. 388 pp.

Li, D., Luong, T. T. M., Dan, W.-J., Ren, Y., Nien, H. X., Zhang, A.-L. and Gao, J.-M. 2018. Natural products as sources of new fungicides (IV): synthesis and biological evaluation of isobutyrophenone analogs as potential inhibitors of classII fructose-1,6-bisphosphate aldolase. Bioorg. Med. Chem. 26:386-393.

Lucas, J. A., Hawkins, N. J. and Fraaije, B. A. 2015. The evolution of fungicide resistance. Adv. Appl. Microbiol. 90:29-92.

Masiello, M., Somma, S., Ghionna, V., Logrieco, A. F. and Moretti, A. 2019. In vitro and in field response of different fungicides against Aspergillus flavus and Fusarium species causing ear rot disease of maize. Toxins (Basel) 11:11.

Miller, N. F., Standish, J. R. and Quesada-Ocampo, L. M. 2020. Sensitivity of Fusarium oxysporum f. sp. niveum to prothioconazole and pydiflumetofen in vitro and efficacy for fusarium wilt management in watermelon. Plant Health Prog. 21:13-18.

Morales, J., Mendoza, L. and Cotoras, M. 2017. Alteration of oxidative phosphorylation as a possible mechanism of the antifungal action of $p$-coumaric acid against Botrytis cinerea. J. Appl. Microbiol. 123:969-976.

Mota, F. L., Queimada, A. J., Pinho, S. P. and Macedo, E. A. 2008. Aqueous solubility of some natural phenolic compounds. Ind. Eng. Chem. Res. 47:5182-5189.

Musso, L., Dallavalle, S., Farina, G. and Burrone, E. 2012. Natural products as sources of new fungicides: synthesis and antifungal activity of zopfiellin analogues. Chem. Biol. Drug. Des. 79:780-789.

Neelam, Khatkar, A. and Sharma, K. K. 2020. Phenylpropanoids and its derivatives: biological activities and its role in food, pharmaceutical and cosmetic industries. Crit. Rev. Food Sci. Nutr. 60:2655-2675.

Nirmaladevi, D., Venkataramana, M., Srivastava, R. K., Uppalapati, S. R., Gupta, V. K., Yli-Mattila, T., Clement Tsui, K. M., Srinivas, C., Niranjana, S. R. and Chandra, N. S. 2016. Molecular phylogeny, pathogenicity and toxigenicity of $\mathrm{Fu}$ sarium oxysporum f. sp. lycopersici. Sci. Rep. 6:21367.

Schneider, S. M., Rosskopf, E. N., Leesch, J. G., Chellemi, D. O., Bull, C. T. and Mazzola, M. 2003. United States Department of Agriculture-Agricultural Research Service research on alternatives to methyl bromide: pre-plant and post-harvest. Pest Manag. Sci. 59:814-826.

Steinkellner, S. and Mammerler, R. 2007. Effect of flavonoids on the development of Fusarium oxysporum f. sp. lycopersici. J. Plant Interact. 2:17-23.

UNEP Ozone Secretariat. 2020. Handbook for the Montreal protocol on substances that deplete the ozone layer. 14th ed. United Nations Environment Programme, Nairobi, Kenya. 937 pp.

Vogt, T. 2010. Phenylpropanoid biosynthesis. Mol. Plant 3:2-20.

Wu, H.-S., Raza, W., Fan, J.-Q., Sun, Y.-G., Bao, W. and Shen, Q.-R. 2008. Cinnamic acid inhibits growth but stimulates production of pathogenesis factors by in vitro cultures of Fusarium oxysporum f.sp. niveum. J. Agric. Food Chem. 56:1316-1321.

Xie, H., Yan, D., Mao, L., Wang, Q., Li, Y., Ouyang, C., Guo, M. and Cao, A. 2015. Evaluation of methyl bromide alternatives efficacy against soil-borne pathogens, nematodes and soil microbial community. PLoS ONE 10:e117980.

Xu, D., Hu, M.-J., Wang, Y.-Q. and Cui, Y.-L. 2019. Antioxidant activities of quercetin and its complexes for medicinal application. Molecules 24:1123.

Ye, S. F., Yu, J. Q., Peng, Y. H., Zheng, J. H. and Zou, L. Y. 2004. Incidence of Fusarium wilt in Cucumis sativus L. is promoted by cinnamic acid, an autotoxin in root exudates. Plant Soil 263:143-150.

Zhao, Q., Chen, L., Dong, K., Dong, Y. and Xiao, J. 2018. Cinnamic acid inhibited growth of faba bean and promoted the incidence of fusarium wilt. Plants (Basel) 7:84. 